\title{
Nonsecretory Multiple Myeloma Presenting as Recurrent Vertebral Compression Fractures in an Older Male Patient
}

\author{
Young Hee Maeng ${ }^{1}$, Chang Lim Hyun ${ }^{1}$, Sang Hoon $\mathrm{Han}^{2}$, Gil Myeong Seong ${ }^{3}$, Dae Hyun Song ${ }^{4}$, Hyo Jung $\mathrm{An}^{5}$, \\ Hyun Min $\mathrm{Koh}^{5}$ \\ ${ }^{1}$ Department of Pathology, Jeju National University School of Medicine, Jeju, Korea \\ ${ }^{2}$ Department of Internal Medicine, Jeju National University School of Medicine, Jeju, Korea \\ ${ }^{3}$ Department of Internal Medicine, Jeju National University Hospital, Jeju, Korea \\ ${ }^{4}$ Department of Pathology, Gyeongsang National University School of Medicine, Jinju, Korea \\ ${ }^{5}$ Department of Pathology, Gyeongsang National University Changwon Hospital, Changwon, Korea
}

Corresponding Author:

Hyun Min Koh, MD, PhD

https://orcid.org/0000-0002-7457-7174

Department of Pathology, Gyeongsang National University Changwon

Hospital, 11, Samjeongja-ro,

Seongsan-gu, Changwon 51472, Korea

Tel: +82-55-214-3151

Fax: +82-55-214-3174

E-mail: shekoh@hanmail.net

Received: January 10, 2018

Revised: March 21, 2018

Accepted: May 30, 2018
Nonsecretory multiple myeloma is a rare variant of multiple myeloma characterized by the proliferation of clonal plasma cells in the bone marrow. It is difficult to establish an early and accurate diagnosis of nonsecretory multiple myeloma because in nonsecretory myeloma cases, monoclonal immunoglobulin cannot be detected in the serum or urine via electrophoresis. In this report, we describe a case of nonsecretory multiple myeloma presenting as recurrent vertebral compression fractures in a 70-year-old male patient and suggest that nonsecretory multiple myeloma can be included in the differential diagnosis of multiple and recurrent vertebral compression fractures, despite the lack of detectable monoclonal immunoglobulin in the serum or urine by electrophoresis. (Ann Geriatr Med Res 2018;22:98-101)

Key Words: Multiple myeloma, Nonsecretory, Vertebral compression fracture

\section{INTRODUCTION}

Multiple myeloma (MM) is a plasma cell disorder characterized by the proliferation of clonal plasma cells in the bone marrow. ${ }^{1)}$ Nonsecretory multiple myeloma (NSMM) is a rare variant of MM first described in the 1950s, which accounts for approximately $1 \%$ to $5 \%$ of myeloma cases. ${ }^{2)}$ NSMM is a symptomatic myeloma characterized by the absence of detectable monoclonal immunoglobulin (Ig) in the serum or urine on electrophoresis. ${ }^{3)}$ Therefore, the diagnosis of NSMM can be delayed and nonspecific symptoms, which often present in patients with NSMM, make the diagnosis more difficult. The case described herein misled doctors by presenting as vertebral compression fractures in an older man with osteopenia.

\section{CASE REPORT}

A 70-year-old man with a past medical history of dyslipidemia was referred to Jeju National University Hospital with a 5-month history of recurrent vertebral compression fractures with back pain. He suddenly had back pain while changing his pants and was treated for T11 and L3 vertebral compression fractures 5 months prior. He had slipped down on the stairs, landing on his backside, and was hospitalized for treatment of L2 and L4 vertebral compression fractures 2 months prior. He had felt back pain
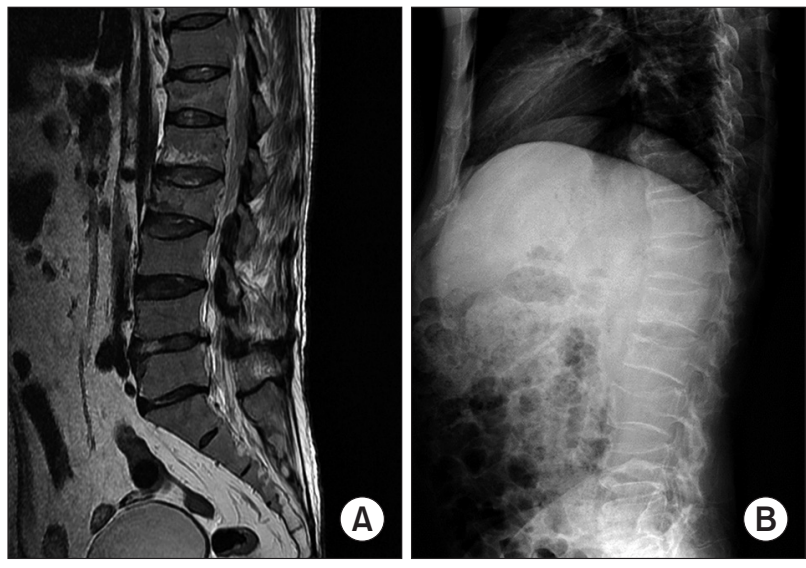

Fig. 1. Magnetic resonance and spine X-ray images. (A) T2weighted magnetic resonance imaging of the lumbar spine reveals vertebral collapse at T12, L1, and L2 with inconspicuous retropulsion of the cortex into the spinal canal. (B) Lateral radiograph of the thoracolumbar spine shows degenerative changes with compression fractures at T12 and the L1 to L3 vertebral bodies. 
while sneezing and had difficulty in walking 5 days prior. Eventually, he was referred to our hospital. Bone densitometry of the spine from previous hospital visits revealed osteopenia, and magnetic resonance imaging (MRI) of the lumbar spine from a previous hospital visit showed multiple compression fractures of the vertebral bodies with inconspicuous retropulsion of the cortex into the spinal canal (Fig. 1A). X-ray images of the T-L spine (Fig. 1B) revealed degenerative changes with compression fractures at T12, and the L1 to L3 vertebral bodies and the pelvic bones (Fig. 2A) showed osteopenia. A bone scan (Fig. 2B) showed a vertebral compression fracture in the L1 spine area; posttraumatic changes in the right first and eighth to tenth ribs and the left sixth rib; and arthritic and degenerative changes in the T9 to L5 spines and both hip joint areas.

Laboratory tests did not reveal specific results. The hemoglobin level was $11.0 \mathrm{~g} / \mathrm{dL}$, showing normocytic normochromic anemia on the peripheral blood smear. We also noted the absence of hypercalcemia $(8.9 \mathrm{mg} / \mathrm{dL})$, and a normal serum creatinine level of $0.78 \mathrm{mg} / \mathrm{dL}$. Labora- tory tests and imaging studies did not provide evidence to suspect that the recurrent compression fractures may have resulted from osteoporosis, metabolic diseases such as hyperparathyroidism, Paget disease and other metabolic processes, or obvious tumors. Therefore, serum and urine protein electrophoresis were conducted for the differential diagnosis of MM. Serum protein electrophoresis did not reveal abnormal Ig levels and the patient's gamma globulin levels were normal. Urine protein electrophoresis showed no monoclonal protein in any significant fraction. Consequently, a presumptive diagnosis of vertebral compression fractures with osteopenia was made, and conservative management was undertaken for 2 weeks.

However, the patient complained of right hip pain during his first ambulation after conservative management. Hip X-ray images (Fig. 3A) suggested several osteolytic lesions, and computed tomography detected multiple inconspicuous osteolytic lesions with greater variegation in opacity than expected in the spine, pelvic bones, both femurs, and ribs, suggesting MM or metastasis (Fig. 3B).
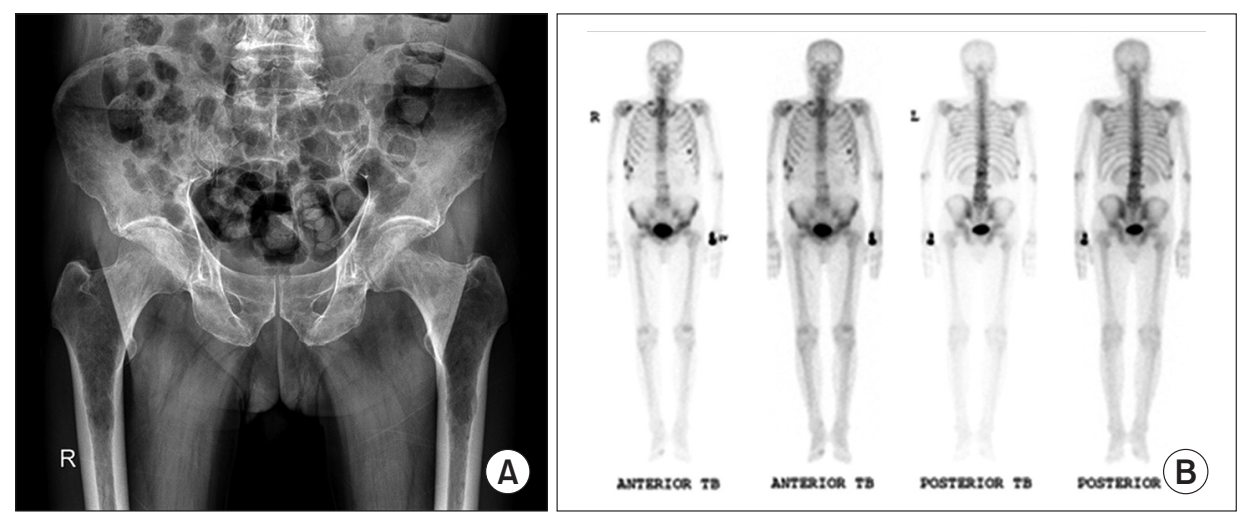

Fig. 2. Pelvic X-ray and bone scan images. (A) Pelvic anteroposterior X-ray image showing osteopenia without conspicuous osteolytic lesions. (B) Bone scan revealing vertebral compression fracture in the L1 spine area with linear and moderate uptake. Posttraumatic changes in the right first and eighth to tenth ribs and the left sixth rib with moderate uptake are also visible. Mild uptake in the T9 to L5 spines and both hip joints area is suggestive of arthritic and degenerative changes.
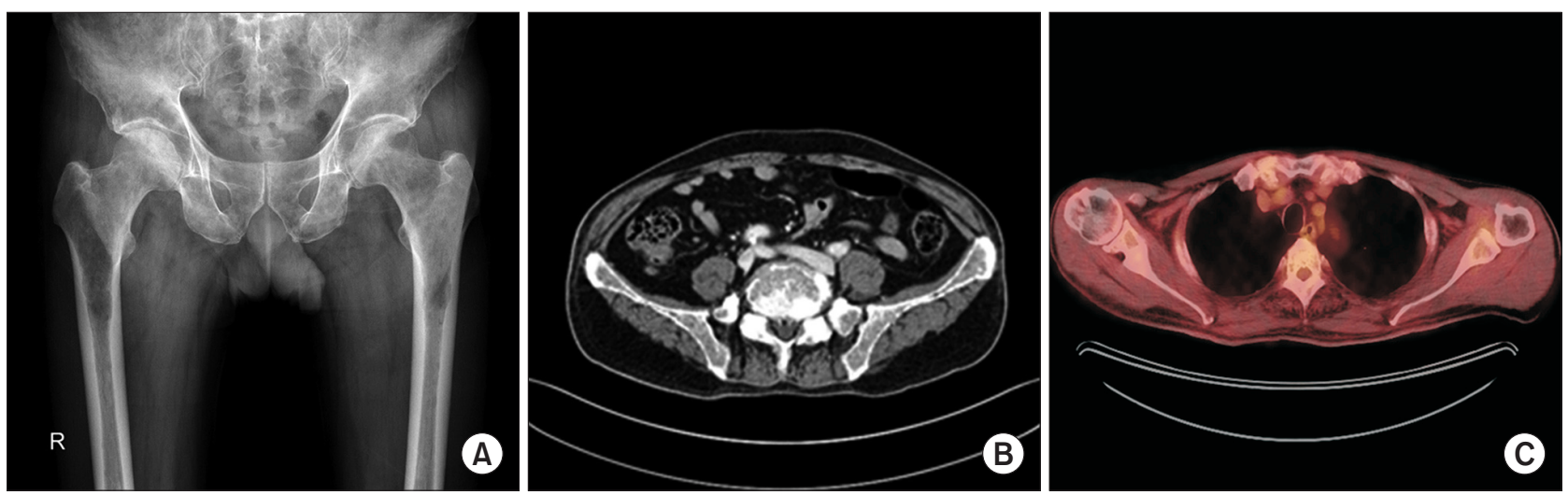

Fig. 3. Hip X-ray, computed tomography, and positron emission tomography images. (A) Hip anteroposterior X-ray image showing several osteolytic lesions in both femurs. (B) Contrast-enhanced computed tomography showing inconspicuous osteolytic lesions with greater variegation in opacity at the vertebral body and pelvic bones. (C) Positron emission tomography revealing a high possibility of malignancy, with significant abnormal 18F-fluorodeoxyglucose uptake in the spine, pelvic bones, ribs, and scapula. 
In addition, positron emission tomography revealed a high possibility of bone metastasis or bone-related malignancy based on a significant abnormal 18F-fluorodeoxyglucose uptake in the skull, spine, sternum, ribs, scapula, pelvic bones, and both femurs (Fig. 3C). Moreover, a serum free light chain (SFLC) assay revealed elevated levels of kappa free light chain $(12.667 \mathrm{mg} / \mathrm{dL})$ and abnormal free light chain ratios with free lambda $(1.105 \mathrm{mg} / \mathrm{dL})$.

Therefore, we performed a bone marrow biopsy for the definitive diagnosis of NSMM. The marrow was mildly to moderately hypercellular and infiltrated by plasmacytoid cells in clusters with some normal hematopoietic cells (Fig. 4A, B). The plasmacytoid cells showed reactivity to CD138 (Fig. 4C) and kappa free light chain on immunohistochemical staining (Fig. 4D), with no reactivity to lambda light chain (Fig. 4E), which supports monoclonal plasma cell proliferation and favors the diagnosis of MM. Finally, the patient was diagnosed with NSMM 7 months since the onset of the first symptom and was referred to the oncology center for further management and therapy.

\section{DISCUSSION}

MM is a common bone marrow-based hematologic malignancy and multifocal plasma cell neoplasm usually associated with the presence of monoclonal Ig in the serum or urine. ${ }^{4,5)}$ The definition of MM from the 2014 International Myeloma Working Group includes 10\% or more clonal plasma cells in the bone marrow or biopsy-proven bony or extramedullary plasmacytoma and one or more of the seven listed criteria (hypercalcemia, renal insufficiency, anemia, bone lesions, clonal bone marrow plasma cells $\geq 60 \%$, involved/uninvolved SFLC ratio $\geq 100$ and involved SFLC concentration $\geq 10 \mathrm{mg} / \mathrm{dL}$, and 2 or more focal lesions based on MRI studies of the skeleton). ${ }^{6}$

The International Myeloma Working Group still defines NSMM as MM lacking monoclonal protein on serum or urine electrophoresis. ${ }^{7)}$ However, this definition is probably not sufficient since an Ig component is actively secreted in MM." Thus, NSMM patients can more accurately be divided into at least 4 groups with separate molecular mechanisms ${ }^{6,7)}$ :

(1) Oligo secretors/FLC-restricted MMs: this tumor is a subset with impaired secretion among tumors with defects in Ig production, which can produce small amounts of light chains. Their protein secretion may not be as high as that seen in typical myeloma, but it can be measured by the SFLC assay. Oligo-secretory $\mathrm{MM}$ is often characterized by SFLC values $<10 \mathrm{mg} / \mathrm{dL}$. Our case is included in this group.

(2) Nonproducers: in these cases, there is complete and real absence of Ig production, resulting in no secretion and no measurable protein in the blood or urine. True nonsecretory myeloma should only be considered this group.

(3) True nonsecretors: these MM cells produce Ig molecules but are unable to secrete them.

(4) False nonsecretors: these are MM variants or related plasma cell diseases with measurable levels of intracellular Ig by immunofluorescence assay but without
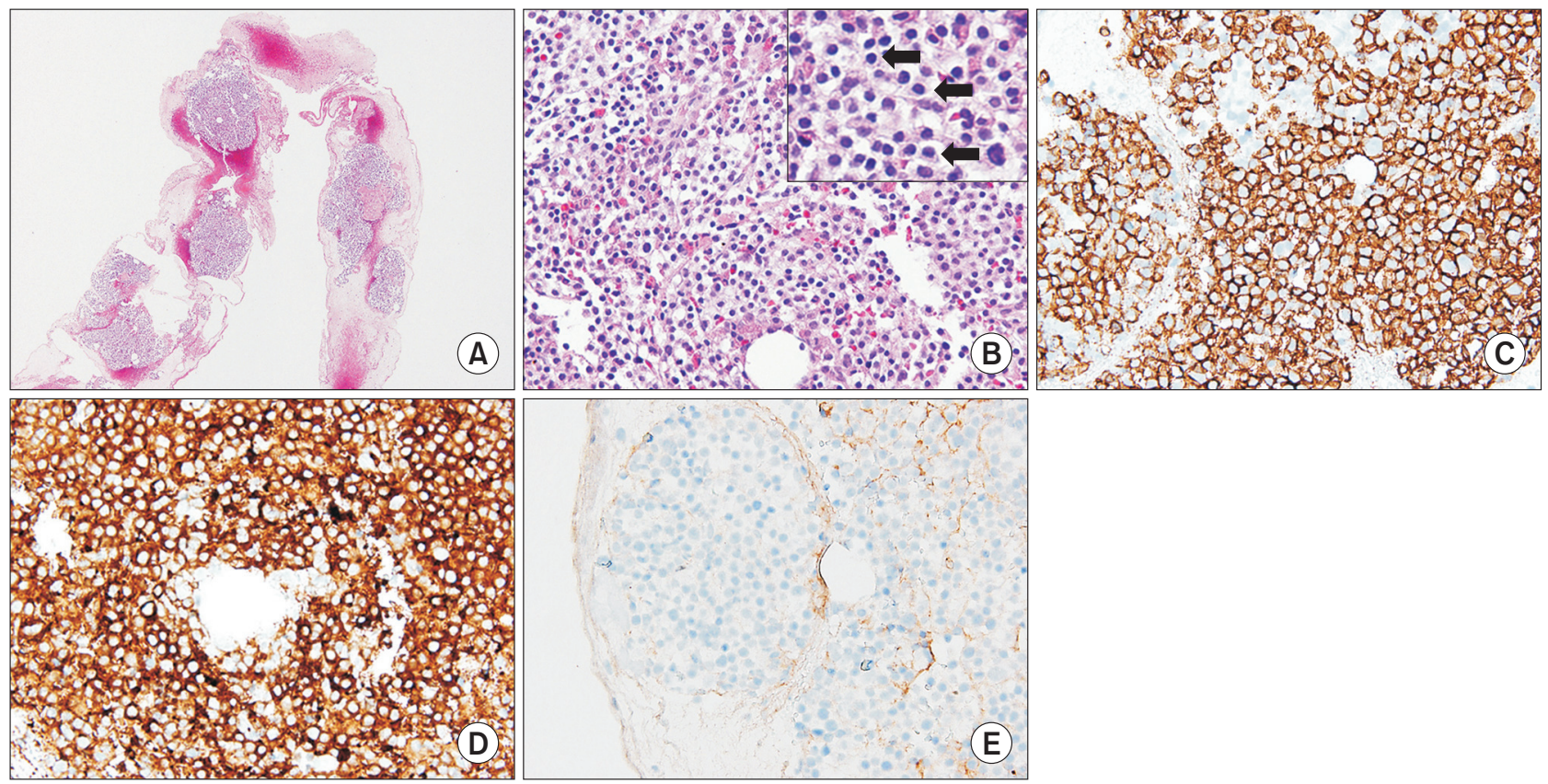

Fig. 4. Bone marrow biopsy. (A) Cellular clusters of bone marrow involvement with plasma cell myeloma (H\&E, $\times 40)$. (B) Plasma cells (black arrows) in clusters with some normal hematopoietic cells (H\&E, $\times 400)$. (C) Tumor cells are reactive to CD138 (immunostaining preparation for CD138, $\times 400$ ). (D) Tumor cells are reactive to kappa light chain (immunostaining preparation for kappa light chain, $\times 400$ ). (E) Tumor cells are non-reactive to lambda light chain (immunostaining preparation for lambda light chain, $\times 400$ ). 
measurable extracellular components by typical testing, despite clear pathologic evidence that they are secreted, such as Ig deposits found in renal biopsies.

NSMM is a rare variant of MM and the clinical features of NSMM are generally similar to those of secretory MM except for a lower incidence of renal insufficiency and hypercalcemia. ${ }^{2,5}$ The clinical course and prognosis of NSMM have not been well studied, since assessment of response and identification of relapse are more difficult given the absence of the monoclonal protein typically used for this purpose in the clinical settings. ${ }^{8)}$ However, based on current studies, NSMM appears to be less aggressive than secretory MM. ${ }^{4)}$ Chawla et al. ${ }^{8)}$ examined the survival and prognosis of 124 NSMM patients in a retrospective study, and the study included SFLC testing where available." They demonstrated that time to progression, progressionfree survival, and overall survival prior to 2001 were similar between NSMM patients and secretory MM patients. ${ }^{4)}$ Between 2001 and 2012, the time frame during which new therapeutics were broadly introduced, there was a marked increase of overall survival in both groups. ${ }^{4)}$ However, the improvement in overall survival was greater in the NSMM group.) In addition, the overall survival was superior in patients with a normal baseline FLC ratio compared to that in patients with an abnormal ratio. ${ }^{8)}$

Vertebral collapse with back pain is a common symptom of MM. However, a delayed diagnosis of MM is not uncommon because vertebral compression fractures are relatively prevalent, especially in elderly with osteoporosis; $10 \%$ to $25 \%$ of patients with MM present with diffuse osteopenia instead of osteolytic lesions, with or without fractures. ${ }^{9)}$ Furthermore, the diagnosis of NSMM with vertebral compression fractures in the elderly is difficult because these patients have no detectable monoclonal proteins in their serum or urine, as discussed above. ${ }^{2,5)}$ Therefore, the diagnosis of NSMM depends on a high suspicion of the disease.

Several case reports have described multiple vertebral fractures in NSMM. These cases showed usually myelomadefining events such as multiple conspicuous osteolytic lesions on MRI or X-ray images, anemia, hypercalcemia, and renal insufficiency. However, our case did not reveal distinct evidence of myeloma on MRI and laboratory tests and also showed vertebral compression fractures with osteopenia on X-ray images of the T-L spine instead of osteolytic lesions. Therefore, we emphasize that recurrent vertebral compression fractures may result from MM if laboratory tests and imaging studies do not provide evidence that the compression fractures have occurred due to osteoporosis, metabolic diseases such as hyperparathyroidism, Paget disease and other metabolic processes, or obvious tumors.

In conclusion, MM can be included in the differential diagnosis of recurrent vertebral compression fractures, despite the lack of detectable monoclonal Ig in the serum or urine by electrophoresis. We recommend bone marrow aspiration, bone marrow biopsy, or biopsy of the osteolytic lesion, SFLC assay and testing for the presence of intracellular Ig by immunofluorescence if NSMM is suspected.

Conflicts of Interest Disclosures: The researchers claim no conflicts of interest.

\section{REFERENCES}

1. Uche E, Akinbami A, John-Olabode S, Dosunmu A, Odesanya M. A rare case of nonsecretory multiple myeloma in Lagos, Nigeria: a case report and literature review. Case Rep Med 2015;2015: 648069.

2. Abdalla IA, Tabbara IA. Nonsecretory multiple myeloma. South Med J 2002;95:761-4.

3. Khurana U, Joshi D, Santoshi JA, Sharma T, Kapoor N. Oligosecretory multiple myeloma: a case report. Blood Res 2016;51:63-5.

4. Dupuis MM, Tuchman SA. Non-secretory multiple myeloma: from biology to clinical management. Onco Targets Ther 2016;9:758390.

5. Elaine SJ, Daniel AA, Elias C, Nancy LH, Leticia QM. Hematopathology, 2nd ed. Philadelphia: Elsevier; 2017.

6. Landgren 0, Rajkumar SV. New developments in diagnosis, prognosis, and assessment of response in multiple myeloma. Clin Cancer Res 2016;22:5428-33.

7. Corso A, Mangiacavalli S. Non-secretory myeloma: ready for a new definition? Mediterr J Hematol Infect Dis 2017;9:e2017053.

8. Chawla SS, Kumar SK, Dispenzieri A, Greenberg AJ, Larson DR, Kyle RA, et al. Clinical course and prognosis of non-secretory multiple myeloma. Eur J Haematol 2015;95:57-64.

9. Derk CT, Sandorfi N. Nonsecretory multiple myeloma masquerading as a new osteoporotic vertebral compression fracture in an older female. J Am Geriatr Soc 2002;50:1747-8. 